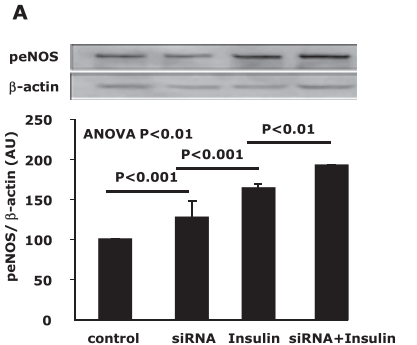

B

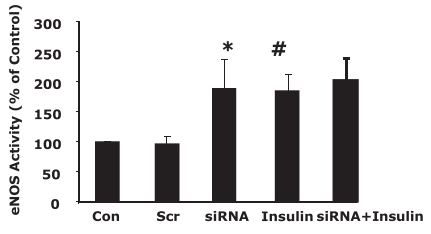

Abstract A Figure 5
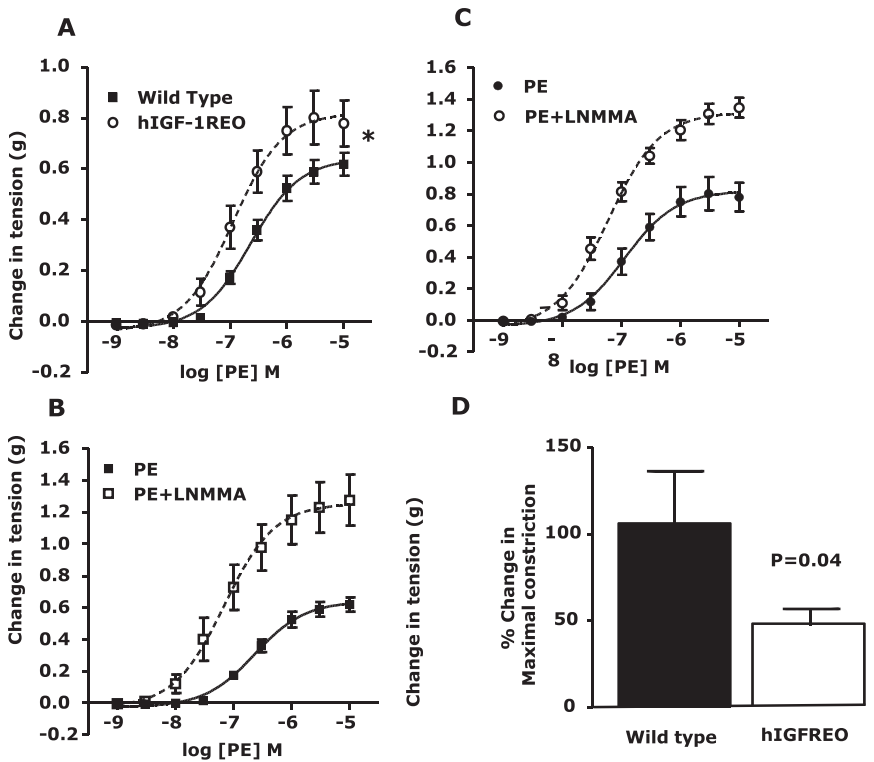

Abstract A Figure 6

strategy by which to modify vascular NO bioavailability and endothelial cell insulin sensitivity.

\section{B VH-IVUS FINDINGS PREDICT MAJOR ADVERSE CARDIOVASCULAR EVENTS. THE VIVA STUDY (VIRTUAL HISTOLOGY INTRAVASCULAR ULTRASOUND IN VULNERABLE ATHEROSCLEROSIS)}

doi:10.1136/heartjnl-2011-300110.2

${ }^{1} \mathrm{P}$ A Calvert, ${ }^{1} \mathrm{D}$ R Obaid, ${ }^{2} \mathrm{~N}$ E J West, ${ }^{2} \mathrm{~L}$ M Shapiro, ${ }^{2} \mathrm{D}$ McNab, ${ }^{2} \mathrm{C} \mathrm{G}$ Densem, ${ }^{2} \mathrm{P}$ M Schofield, ${ }^{2} \mathrm{D}$ Braganza, ${ }^{2} \mathrm{~S} \mathrm{C}$ Clarke, ${ }^{2} \mathrm{M}$ O'Sullivan, ${ }^{3} \mathrm{~K}$ K Ray, ${ }^{1} \mathrm{M}$ R Bennett. ${ }^{1}$ University of Cambridge, Cambridge, UK; ${ }^{2}$ Papworth Hospital NHS Foundation Trust, Cambridge, UK: ${ }^{3}$ St George's University of London, London, UK

Background Identification of high-risk atherosclerotic plaques offers opportunities for risk stratification and targeted intensive treatment of patients with coronary artery disease. Virtual Histology intravascular ultrasound (VH-IVUS) has been validated in human atherectomy and post-mortem studies and can classify plaques into presumed high- and low-risk groups. However, VH-IVUS-defined plaques have not been shown to be associated with major adverse cardiovascular events (MACE), or biomarkers that confer increased cardiovascular risk, such as serum cytokines or shortened leukocyte telomere length (LTL)

Methods 170 patients with stable angina or troponin-positive acute coronary syndrome (ACS), referred for percutaneous coronary intervention (PCI) were prospectively enrolled and underwent full 3-vessel VH-IVUS pre-PCI. Troponin-I (cTnI), IL-6, IL-18, hsCRP, neopterin, MCP-1 and sICAM-1 were measured pre-PCI and 24-h post-PCI. LTL was determined by qPCR. The combined primary endpoint (MACE) included unplanned revascularisation, myocardial infarction (MI) and death, with a secondary endpoint of post-PCI MI (MI 4a).

Results 18 MACE occurred in 16 patients (median follow-up: 625 (463-990) days). $30372 \mathrm{~mm}$ of VH-IVUS were analysed and 1106 plaques classified (Abstract B Figure 1) locally and via a core-lab. After multivariable regression:

1. Total number of non-calcified VH-IVUS-identified thin capped fibroatheromata (VHTCFA) was the only factor independently associated with MACE ( $\mathrm{HR}=3.16$, (95\% $\mathrm{CI}=1.16$ to 8.64$), \mathrm{p}=0.025)$.

2. Total VHTCFA number $(\mathrm{OR}=1.26(1.03$ to 1.53$) \mathrm{p}=0.021)$ and total stent length $(\mathrm{OR}=1.04$ (1.01 to 1.08$), \mathrm{p}=0.01)$ were the only factors independently associated with MI 4a.

3. A novel 3-vessel vulnerability index (necrotic core: fibrous tissue ratio) and side branch loss were independently associated with stenting-related $\mathrm{cTnI}$ rise (standardised beta coefficient $(s \beta)=0.29, p=0.004$ and $s \beta=0.23, p=0.019$ respectively).

4. Necrotic core area at the minimum luminal area frame was the only factor independently associated with ACS presentation $(\mathrm{OR}=1.59, \mathrm{p}=0.030)$.

5. Stented vessel VHTCFA number $(\mathrm{OR}=1.75$ (1.22 to 2.51), $\mathrm{p}=0.002)$ was independently associated with the lower LTL tertile (DNA-based cardiovascular risk predictor).

6. Stenting-related IL-6 rise was the only biomarker independently associated with MACE ( $\mathrm{HR}=1.03 \quad(1.01-1.05)$, $\mathrm{p}=0.007$ ).

Conclusion We present the first report of an association between VHTCFA and MACE. This provides novel evidence that VHTCFA definitions are important in their own right (rather than as analogues of histological TFCA definitions). We also present the first report of associations between VHTCFA and MI $4 \mathrm{a}$ as well as a novel vulnerability index that is association with stenting-related troponin rise. Finally, we report a novel association between VHTCFA and DNA-based cardiovascular risk prediction (LTL)

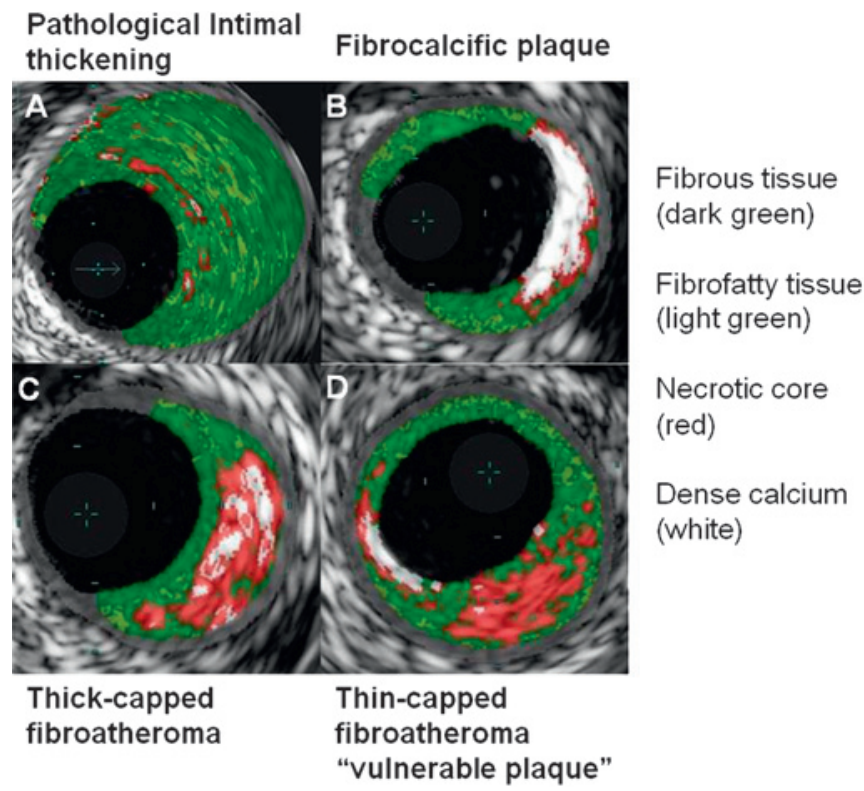

Abstract B Figure 1 